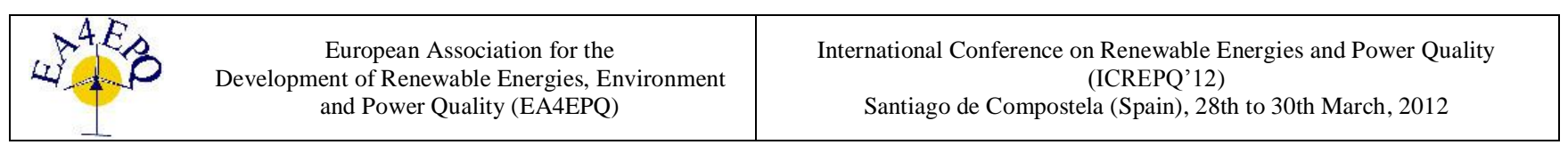

\title{
Survey of Inductance Curves in Switched Reluctance Machines Using Finite Elements
}

\author{
M. B. Rego, L. C. Gomes, A. B. F. Neves, A. W. F. V. Silveira and E. A. A. Coelho \\ Laboratory of Electrical Drives (L.A.C.E.) \\ F.E.E.L.T., Universidade Federal de Uberlândia \\ Av. João Naves de Ávila, 2121, 38400-299 Uberlândia, Brazil \\ e-mail: mariana.barbosarego@gmail.com
}

\begin{abstract}
The aim of this document is combine theoretical knowledge, computational tools and mathematical analysis so that the curves of inductance of two topologies for switched reluctance machines can be studied and analyzed. This article shows how to obtain the inductance curves for many values of current, using a magnetic simulation software named FEMM (Finite Elements Method Magnetics) and the programming language Lua script. The data analyses are to the $6 \times 4$ machine, three phase, and the $6 \times 6$ machine, single phase. Lastly, the computational simulations are compared with the real simulations made with the machines available in the university's research laboratory.
\end{abstract}

\section{Key words}

Inductance Curves, FEMM, Lua Script, Switched Reluctance Machines.

\section{Introduction}

The motivation for the study of the switched reluctance machine (SRM) is based on technical and economic parameters. Currently, the SRMs have a great potential to be used in drive applications because, besides being sturdier and lighter compared to other machines with similar power outputs, they have relatively simple construction and lower manufacturing costs. The most notable SRMs features, which also differentiate them from others, are the fact that there are no permanent magnets or windings on the rotor and, also, it is needed only one excitation source applied to the stator winding to move it [1-4].

Considering all the above advantages, this work consists in developing a program that raises the inductance curves of switched reluctance machines using the FEMM software and Lua script programming language.
FEMM is a freely distributed software, used to solve lowfrequency electromagnetic problems in two dimensions and at planar and axisymmetric domains, using for this purpose, finite element analysis and definition of boundary conditions [5].

This software is divided into three basic parts. The first is the executable itself FEMM.exe called Interactive Shell. At this part the user can deal with all the variables to preand post-process the problem. The second part, called triangle.exe, treats the problem of fragmentation into triangles. Lastly, the third part, designated of fkern.exe to magnetic problems, solves the problem [5].

FEMM is divided into definition of problems of preprocessing, processing and post-processing: the machines parameters are defined at pre-processing, the calculations are made at processing and, finally, the results and calculated data can be observed at post-processing.

To automate the process of simulation, we used the programming language Lua script. This is an open source language used to create scripts that are used by FEMM software to make the resolution of some cases simpler. With this program the user can set the whole simulation process, what means that the user can create the geometry of the problem and define the materials to obtain data to draw the curves of inductance [5].

Using FEMM, the simulation of two SRM topologies were made: a three-phase, with six stator poles and four rotor poles $(6 \mathrm{x} 4)$, and a single phase, with six stator poles and six rotor poles $(6 \times 6)$. Through these simulations a database was created, in a way the curves of inductance could be traced for several values of current and rotor positions.

From the curve, the mathematical simulation of the machine was optimized and the results were very close to the experimental. 


\section{Switched Reluctance Machines}

\section{A. Theoretical Introduction}

Basically, a SRM rotor moves in order to find the position where the reluctance is minimum and the inductance is maximum. The torque is produced by the tendency of the rotor to align itself with the flux wave produced by the stator in order to maximize the flux linkage, which is a result of applying a current in the stator. For the same current value, the more the flux linkage is increased, the more the value of inductance will be increased. The flux linkage varies with the position of the rotor and is maximum when the rotor axis is aligned with the magnetic axis of phase [6].

The survey of magnetization curves in the SRM needs a computational analysis and it must consider the partial effect of concatenation, which states that for some positions of the rotor windings, the coil does not concatenate the same stream. For intermediate positions of the rotor alignment, the calculations of magnetization curves are not made in a practical way by the analytical method, to do so, it is necessary to use the finite element method [7].

The finite element method can be understood as a numerical approximation to solve problems that are not easily solved in an analytical way. In simple terms, it can be said that the finite element method reduces a complex problem in a number of problems with simpler solutions [8].

In this context, the FEMM minimizes the problem domain using triangular elements. For each of these elements, the solution is approximated by a linear interpolation of potential values obtained at each vertex of the triangle. These approximations are made to minimize the error between the solution of differential equations and the approximated solution by finite element method [5].

\section{B. Utilized Machines}

To develop this study two switched reluctance machines were used. They were specially designed for studies and research projects at the Laboratory of Electrical Drives (LACE) at the Federal University of Uberlandia (UFU). They are a $6 \times 4$ and a $6 \times 6$ machine.

The machines are designed to provide a power output of approximately $6 \mathrm{CV}$ at the shaft, with a rated speed of 1200 revolutions per minute and 40 turns per tooth. Figures 1 and 2 show cross sections of both SRMs, where is possible to see the teeth of the rotor and the stator and a part of the winding of phase A.

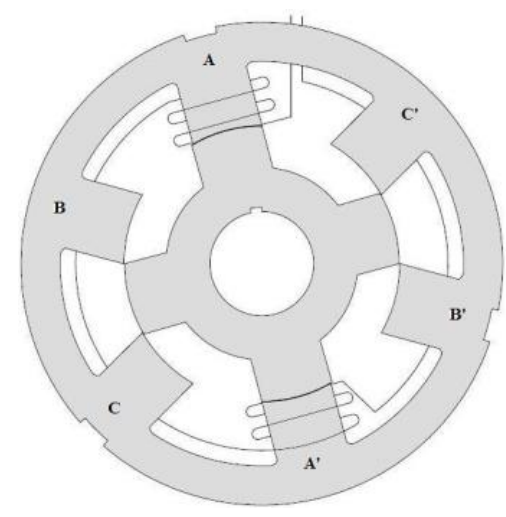

Fig. 1. Cross section of the $6 \times 4$ SRM

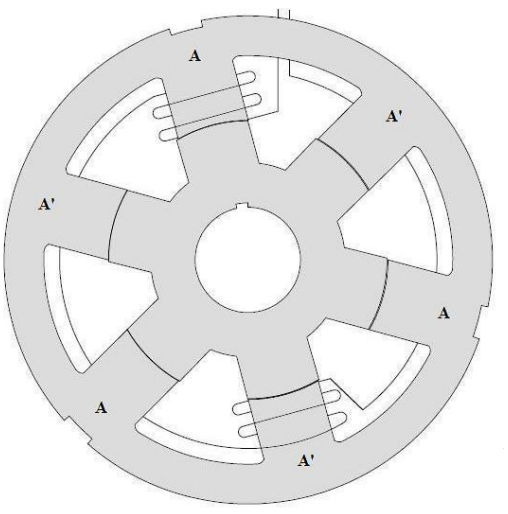

Fig. 2. Cross section of the $6 \times 6$ SRM

\section{Survey of Inductance Curves}

To obtain the lifting of the inductance curves were defined 54 current values for the simulation curves for each position of the rotor on both machines. The values are in the range of $2 \mathrm{~A}$ to $30 \mathrm{~A}$, ranging from $0.5 \mathrm{~A}$ to 0.5 A. The simulation collect for each position of rotor and for each value of current the flow values related. So, was necessary vary the rotor position from 1 on 1 and the current values after each complete cycle of the machine. In the $6 \times 4$ machine simulations were made ranging from $0^{\circ}$ to $45^{\circ}$ and in $6 \times 6$ ranging from $0^{\circ}$ to $30^{\circ}$. That means that 2668 simulations were made to the $6 \times 4$ machine and 1798 for the $6 \times 6$ machine, a total of 4466 different simulations.

For the development of simulations, were generated lists relating current values, rotor position and flux linkage, through a program code in the Lua language. From these data, it was possible to trace the behavior of the inductance for each current applied in machines and for every angle developed by the rotor. The data simulated by the software were compared with the data obtained in real time simulations, performed at the Laboratory of Electric Drives, with the machines described in Section 2.B.

To trace the curves of inductance, virtual spreadsheets got the point values of inductance for each angle that defined the rotor position for a given amount of current. 54 charts were made for each of the machines. 


\section{A. Setting Parameters in FEMM}

In FEMM parameters of pre-processing the user can graphically express the problem and define the boundary conditions and materials to be used. At this study, the cuts of the rotor and stator of the machines, were designed with the aid of a CAD software and, after this, the images were imported to FEMM. Then the materials used in the machine, the circuits, the number of windings, the boundary conditions of the problem and the rotor position were defined.

For the axis of the machine was set to use 1020 carbon steel, for the rotor and the stator, laminated silicon steel M22 and 10AWG wire windings were chosen. Since both machines have six poles in the stator, were established for each one, three reels with 40 windings. After that, begins the processing step. First, a triangulation is made, splitting the problem into thousands of tiny triangles to make possible the analysis by finite elements. Figure 3 shows part of one fragmented machine divided into triangles. Now the simulation is performed and, subsequently, the results can be seen.

In the post-processing, the software has a new interface and a new file format. In this new interface the user can view several types of curves and get some parameters such as torque, magnetic field, power, voltage drop, etc. The software makes possible to obtain estimated points of the value of inductance for each current and rotor position simulated.

To exemplify the utilization of FEMM, the figure 4 shows the use of post-processing, in which the magnetic flux density is displayed according to the concentration of the lines. In Figure 4, the darker parts and more lines drawn, denotes highest levels of flow and the lightest and low concentrations of lines, lower intensities of flow.

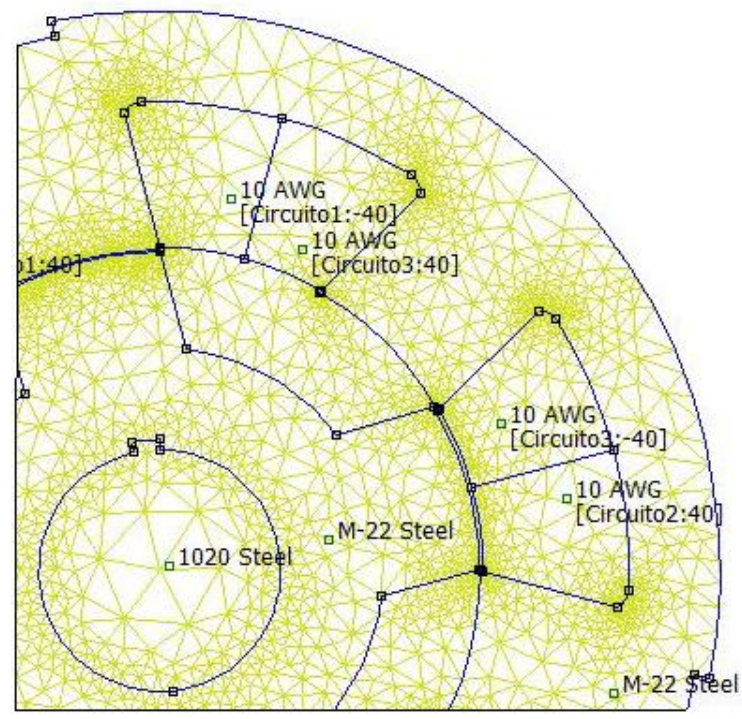

Fig. 3. Triangles traced by FEMM to make the finite element analysis (SRM 6x4)

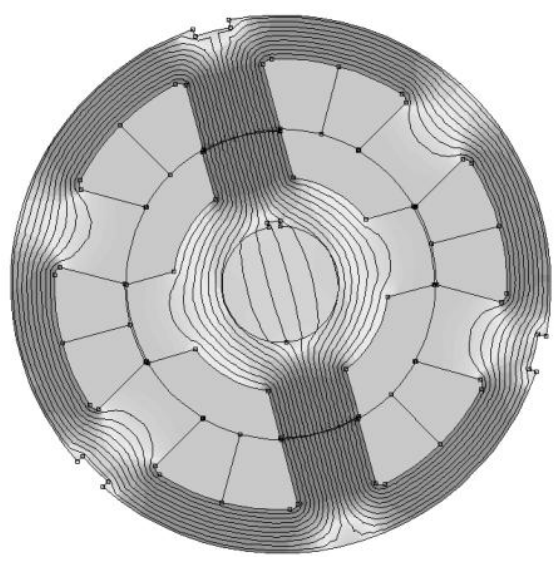

Fig. 4. Magnetic flux density (SRM 6x4)

\section{B. Programming with Lua}

As described above, the possibility of using Lua in the development of this project was crucial to the success in obtaining the curves of inductance. Would be very tough perform the entire process of positioning the rotor with their current values and redefining the parameters of preand post-processing by the user each time, due to the huge amount of simulations. Thus, the following code using Lua script automates the whole process.

The code developed consists in using interactive loops. First we define the loop that makes the current vary from $2 \mathrm{~A}$ to $30 \mathrm{~A}$ and, within this loop, another loop that defines the rotor position with a variation from $1^{\circ}$ to $1^{\circ}$. It means that for each current the program stores the values of inductance for many rotor positions. For better understanding, Figure 5 shows the code developed for the $6 \times 4$ machine.

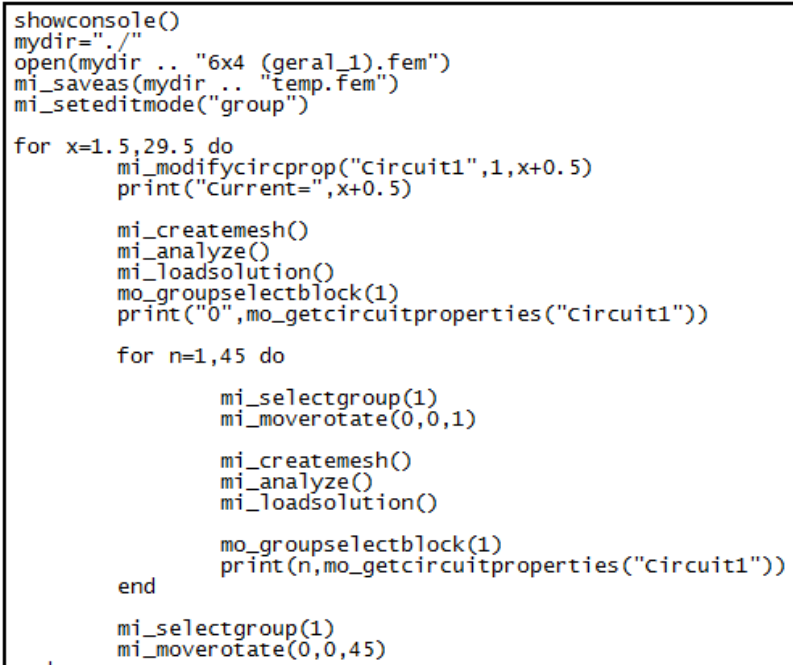

Fig. 5. Using Lua script to generate the inductance curve automatically

As the program executes the script, the data of flux linkage, voltage, current and the rotor position are obtained and stored. With this information it is possible to calculate the inductance value by the relation between 
current and flow. The average processing time for each of the codes was approximately 5 hours.

\section{Results Obtained}

\section{A. Profiles of inductance paths}

As mentioned previously, the data obtained by simulations with the software FEMM were turned into tables and, for some values of current, the inductance curve obtained by collecting data in real machines could be faced with curves obtained computationally. The survey of the experimental curve of inductance is limited by the power supply, so it did not exceed the value of $15 \mathrm{~A}$. Using the software, simulations were made until a current of $30 \mathrm{~A}$, that gives a preview of the magnetic saturation effect. To exemplify, figure 6 shows a plot for SRM 6x6 applying a current of 6A. In total, 108 charts were drawn. Figure 6 shows the relationship between the values of inductance and rotor position, for the practical simulation (dotted line) and the computer simulation, obtained in FEMM (solid line). The discrepancies found may be justified because of the way the experimental values of the inductance were obtained. The rms current and voltage at the terminals of the machine were measured and applied at the formula (1). The inductance values ware calculated knowing that the machine is connected to the network, which has a frequency of $60 \mathrm{hz}$, and knowing the resistance value of the machine. It is known that the calculation of inductance using this formula is valid only for application of a purely sinusoidal voltage at the machine terminals. The test was done by connecting to the network machine, so this calculation had an approximate value, because the voltage in the network is not purely sinusoidal, harmonic features are added to it.

\section{B. Relation between current, inductance and rotor position}

The development of this work involved getting a lot of data, graphs and tables, which make the analysis of the results more comprehensive. To deal this amount of data were used the MATLAB ${ }^{\circledR}$ software. The values were related to current, inductance and rotor position in two three-dimensional graphs. Figures 7 and 8 show the curves of inductance as a function of current and rotor position. It is seen that for current values from $20 \mathrm{~A}$, the variation of the inductance is much lower, indicating, therefore, the saturation of magnetic material. This data is very important because it shows the physical limits for each of the machines, indicating that after this current value, the flux linkage does not fluctuate much, despite the performance of the machine is reduced.

$$
L=\frac{\sqrt{\frac{V^{2}}{I}-R^{2}}}{\omega}
$$

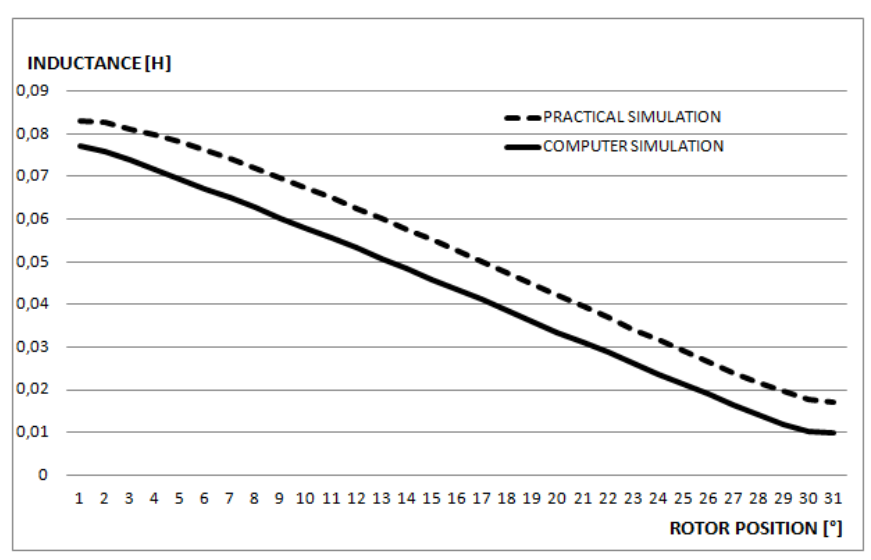

Fig. 6. Chart of the inductance curves for experimental and simulated computationally SRM 6x6 in current $6 \mathrm{~A}$

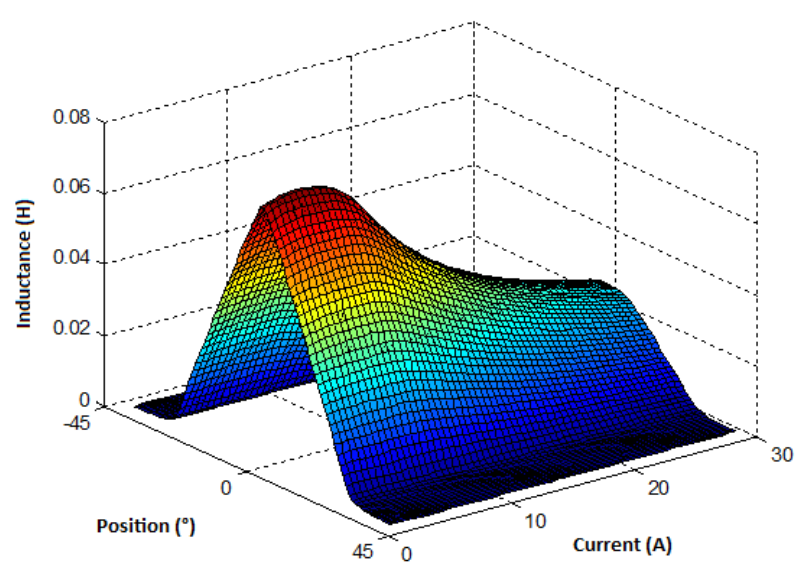

Fig. 7. Inductance curve (SRM 6x4)

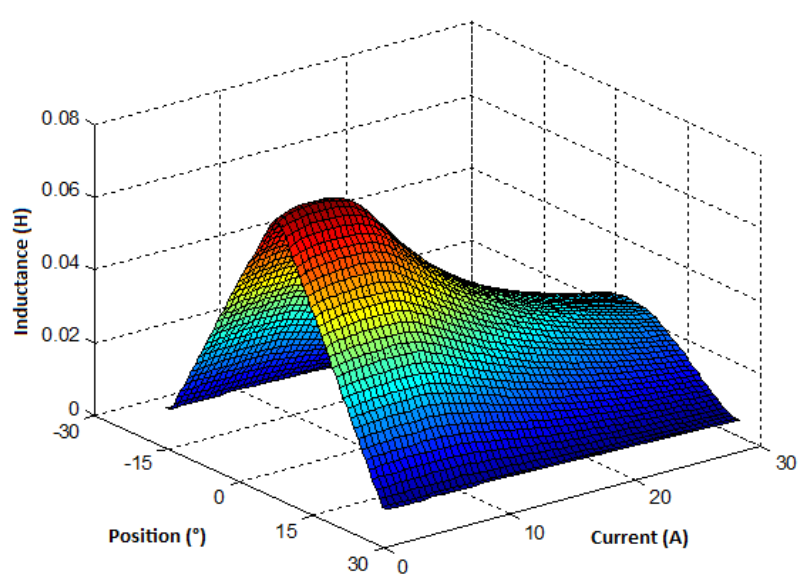

Fig. 8. Inductance curve (SRM 6x6)

\section{Experimental Results}

A comparison was made between the experimental results and those obtained in the simulations. Figures 9 and 10 show the waveforms of current in phase "a" of SRMs. As can be seen, the shape and amplitude of the current trial are quite compatible with the current waveform simulation. That means that the inductance curve generated by finite element software, FEMM, gave results very close to reality, and quite satisfactory. Analyzing the figures 9 and 10 it is clear that the simulated results are very close to those obtained 
experimentally, both in amplitude and wave form. That shows that the simulation provided very reliable data to the study of the switched reluctance machines.

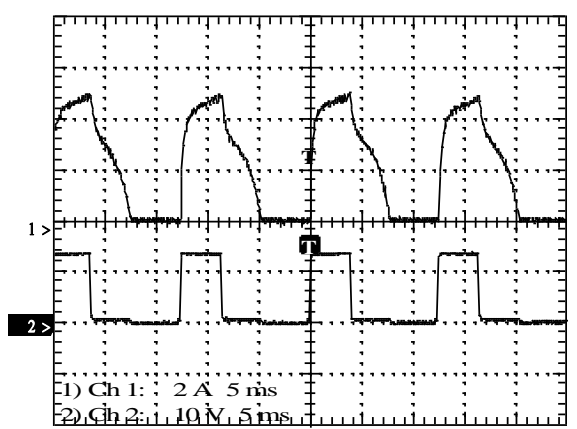

(a)

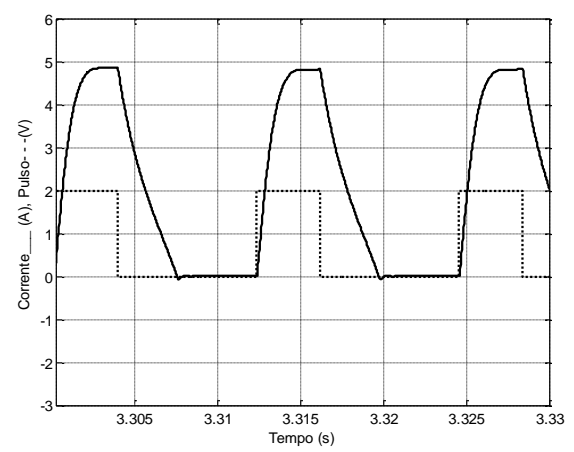

(b)

Fig. 9. Current and trigger during the three phases of SRM. (a) experimental and (b) simulated

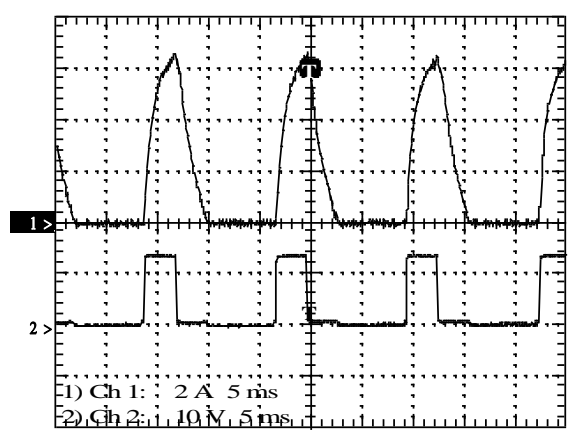

(a)

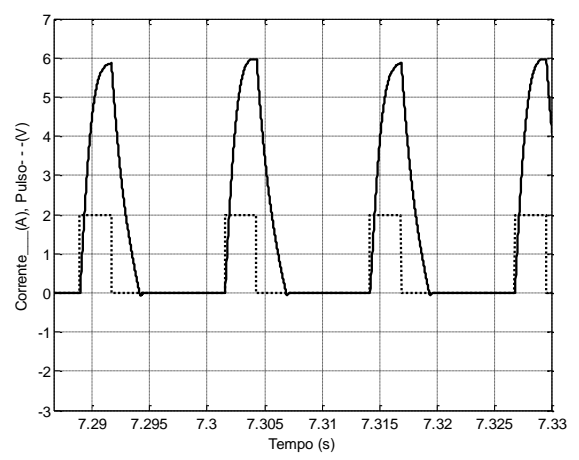

(b)

Fig. 10. Current and trigger the phase of single-phase SRM. (a) experimental and (b) simulated

\section{Conclusions}

This work has shown a study of a problem using the simulation program of finite elements (FEMM) to obtain the curves of two inductance topologies of the switched reluctance machines: a three-phase, $6 \times 4$, and a single phase, 6x6. Were also outlined the necessary steps for the development of a computer simulation using the FEMM software and the Lua script code. The realization of this code has automated the curves of inductance for each of the machines. It was verified, by comparing experimental results with simulated results, that the curve of inductance created by the software comes up quite accurately to the experimental curve. This is very important because it saves time and resources that would be demanded in obtaining an experimental flow curve.

\section{Acknowledgement}

The authors thank FAPEMIG and CNPq for financial support to this study.

\section{References}

[1] H.C. Lovatt, M. L. McClelland, J.M. Stephenson, "Comparative Performance of Singly Salient Reluctance, Switched Reluctance, and Induction Motors", IEE EMD97, no. 444, pp361-365, 1-3 September 1997.

[2] M. J. Turner, P. Y. P. Wung, R. S. Wallace, "Comparing European 132 Frame Switched Reluctance and Induction Motor Drives", pp403-408.

[3] H. Moghbelli, G. E. Adams, R. G. Hoft, "Performance of a 10-Hp Switched Reluctance Motor and Comparison with Induction Motors", IEEE Transactions On Industry Applications, vol. 21, no. 3, pp531-538, May/June 1991.

[4] M. A. Mueller, "Design and Performance of a 20Kw, $100 \mathrm{rpm}$, Switched Reluctance Generator for a Direct Drive Wind Energy Converter", IEEE International Conference on Electric Machines and Drives, 2005.

[5] D. Meeker (2009). "Finite Element Method Magnetics: Version 4.2, User's Manual”. Accessed in $24^{\text {th }} \quad$ August, 2010, at: <http://www.FEMM.info/Archives/doc/manual42.pdf>.

[6] A. E. Fitzgerald, C. Kingslay Jr., S. D. Umans, "Electric Machinery", Mc Graw Hill, 6 ${ }^{\mathrm{a}}$ Edition, New York, 2003.

[7] T. J. E. Miller, "Speeds Electric Machines: An outline of some of the theory in the speed software for electric machine design with problems and solutions", University of Glassgow, 2002-2009.

[8] K. H. Huebner et al., "The Finite Element Method for Engineers", John Wiley \& Sons, $4^{\text {a }}$ Ed., New York, 2001. 